\title{
DÜBLIN
}

Technological University Dublin

ARROW@TU Dublin

\section{Facilitating smart HACCP strategies with Process Analytical Technology}

\author{
Fintan Moran \\ Technological University Dublin, fintan.moran@tudublin.ie \\ Carl Sullivan \\ Technological University Dublin, carl.sullivan@tudublin.ie \\ Kevin Keener \\ lowa State University
}

See next page for additional authors

Follow this and additional works at: https://arrow.tudublin.ie/schfsehart

Part of the Food Science Commons

\section{Recommended Citation}

Fintan Moran, Carl Sullivan, Kevin Keener, Patrick Cullen, Facilitating smart HACCP strategies with Process Analytical Technology, Current Opinion in Food Science, Volume 17, 2017, Pages 94-99, ISSN 2214-7993, DOI: 10.1016/j.cofs.2017.11.003.

This Article is brought to you for free and open access by the School of Food Science and Environmental Health at ARROW@TU Dublin. It has been accepted for inclusion in Articles by an authorized administrator of ARROW@TU Dublin. For more information, please contact arrow.admin@tudublin.ie, aisling.coyne@tudublin.ie, gerard.connolly@tudublin.ie.

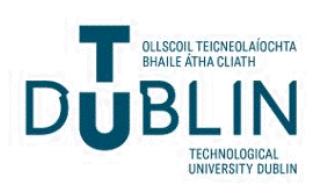




\section{Authors}

Fintan Moran, Carl Sullivan, Kevin Keener, and Patrick J. Cullen

This article is available at ARROW@TU Dublin: https://arrow.tudublin.ie/schfsehart/465 


\section{Facilitating smart HACCP strategies with Process Analytical Technology Fintan Moran ${ }^{1}$, Carl Sullivan ${ }^{1}$, Kevin Keener ${ }^{2}$ and Patrick Cullen ${ }^{1,3}$}

The critical feature to any food management system, be it safety or quality focused, is timely and accurate product and process data. The HACCP framework is one of a systematic preventive approach that addresses hazards as a means of prevention rather than finished product inspection. The PAT initiative is a framework which also aims to change product assurance from end-product testing to preventive control through timely measurements of critical product and process parameters. The continued emergence of sensitive yet affordable sensors capable of direct measurement of biological, chemical and physical risks associated with foods during production, processing or over their shelf-life undoubtedly will support a higher temporal and spatial profiling of foods.

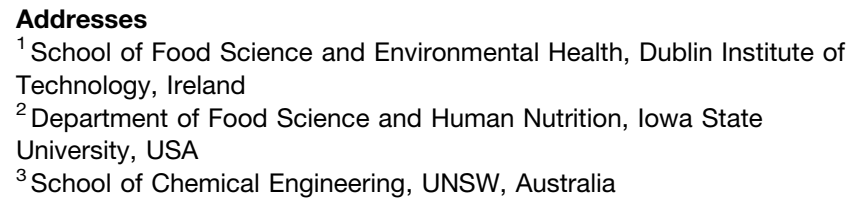

${ }^{3}$ School of Chemical Engineering, UNSW, Australia

Corresponding author: Cullen, Patrick (pjcullen@dit.ie)

Current Opinion in Food Science 2017, 17:94-99

This review comes from a themed issue on Food engineering and processing

Edited by Colette Fagan

For a complete overview see the Issue

Available online 8th November 2017

http://dx.doi.org/10.1016/j.cofs.2017.11.003

2214-7993/@ 2017 Elsevier Ltd. All rights reserved.

\section{Introduction \\ HACCP}

The Hazard Analysis and Critical Control Point (HACCP) approach to food safety is the most important food safety management system globally, where food safety is addressed through analysis and control of biological, chemical and physical hazards. The framework is one of a systematic and preventive approach to food safety that addresses hazards as a means of prevention rather than sole reliance on finished product inspection. The strategy is applicable over the entire food chain.
HACCP changes the emphasises from end-product testing to preventive control of critical aspects of producing safe foods. Since its development in the 1960s, HACCP principles have been defined and endorsed in international food standards (Codex Alimentarius Commission), becoming a universally accepted methodology for food safety assurance with its impact on global food safety clear. The success of the approach is due to the flexibility of the system allowing it to apply to the diverse nature of food commodities, the extremes of scales of food production and the many processing techniques encountered in the food industry.

The seven principles of HACCP are part of the international standard: ISO22000 FSMS 2005, a complete food safety and quality management system which accounts for both HACCP and its prerequisite programs including; Good Manufacturing Practices (GMP), Sanitation Standard Operating Procedures (SSPO). Together these units form a Total Quality Management (TQM) system. HAPPC may be integrated with hierarchical quality management systems such as ISO900 or equally with innovative monitoring and data management strategies. Key to the success of the approach is a detailed understanding of the food product, process conditions and environment factors that ultimately govern the safety profile of the product [1]. With the development of affordable and non-invasive sensors opportunities have emerged to capture and use high resolution real-time data directly from the production line, transport and storage to both characterise and control products during production and shelflife $\left[2^{\bullet \bullet}\right]$.

\section{Process Analytical Technology initiative (PAT)}

The PAT initiative is a framework for innovative process manufacturing and quality assurance. The approach is to design, analyse, and control manufacturing through the timely measurement of identified critical control parameters which govern product variability. The identified benefits of the framework include increased process efficiency, reduced operating costs, increased process validation and ultimately improved final product quality and safety. As with HACCP, the framework aims to change the product assurance from end-product testing to preventive control of critical parameters.

The modern period of Process analytical chemistry (PAC) essentially began with the formation of the Centre for 
Process Analytical Chemistry (CPAC) in 1984 [30] which defined five "eras" of PAC: (1) off line, (2) at line, (3) online (4) inline, and (5) non-invasive, which describe the evolution of sensor technologies [4]. Its definition has evolved over the years to encompass analytical measurements and understating of chemical, physical, microbiological parameters governing processing. Changing the term "Chemistry" to "Technology" allowed a broader scope of the approach to other processes. 'Analytical' has emerged to include; chemical, physical, microbiological, mathematical, and risk analysis parameters and methodologies.

The objective of this paper is to introduce the PAT framework and discuss the potential impact such data rich approaches may have on the HACCP food safety management system.

\section{Potential implementation of PAT to the 7 HACCP steps \\ Principle 1: Hazard analysis}

Principle 1 concerns the process of collecting and evaluating information on hazards and conditions leading to their presence to decide which are significant to food safety. Quality by Design (QbD) is a manufacturing concept initially presented by Joseph Juran [5]. Within the pharma sector QbD is defined as 'a systematic approach to development that begins with predefined objectives and emphasizes product and process understanding and process control, based on sound science and quality risk management' (ICH [6]). Risk assessment and management, raw material management, use of statistical approaches and PAT provide a foundation to these activities [ $\left.7^{\circ}\right]$. It is apparent that $\mathrm{QbD}$ approaches have a clear role in a more multi-factorial analysis of such a hazard over those typically employed.

Certain foods such as infant formula, will have nutritional composition as a critical safety factor and mirror solid dose pharmaceuticals in terms of dose risk. With regards to biological risk assessment, there is no 'safety by design' initiative however extending the design to understand the role of physical properties such as ingredient particle size distributions and particle porosity on moisture content and potential microbial growth will provide a more integrated analysis of such hazards and ultimately improved preventative strategies. Data for such multifactorial hazard analysis are typically available for food hazards but rarely employed for establishing critical control points for typical industrial adoption of HACCP. An advantage of PAT sensors is the ability to carry out such multifactorial analysis in the lab followed by integration of the same sensor into the process line.

\section{Principle 2: Critical Control Point (CCP)}

A CCP is a point, step, or procedure in a food manufacturing process at which control can be applied and a food safety hazard can be prevented, eliminated, or reduced to an acceptable level. Within the confines of PAT a process parameter whose variability has an impact on a on a critical quality attribute is termed a Critical Process Parameter (CPP). Capturing data on the variability of identified CCPs could facilitate a smarter approach to controlling parameters where the process learns from its past performance. HACCP CCPs have traditionally been univariate in nature and minimally consider the variable nature of raw materials, products, processes or microbes etc. PAT provides information from the processing line continually building data on variables, both product and process, which may govern a CCP and in essence lead to continuous process validation.

\section{Principle 3: Establish critical limits}

A critical limit is the maximum or minimum value to which a physical, biological, or chemical hazard must be controlled to prevent, eliminate, or reduce to an acceptable level. As a control strategy, PAT employs such limits to establish corrective actions and control. However, the provision of such large data sets from real-time monitoring may identify product or processes which are briefly outside of the critical limits for example temporal-spatial fluctuations during processing. Consequently, approaches such as moving averages may be employed to filter such occurrences. Similarly establishing critical limits for HACCP has typically assumed univariate values although the risk may indeed be multivariate. This has often led to over processing of foods to ensure product safety which in turn can lead to avoidable detrimental quality or nutritional affects. Greater insights into CCPs and smarter control strategies could facilitate not only improved safety systems but also help limit over processing.

\section{Principle 4: Establish system to monitor CCP}

Process or product monitoring is required to ensure that the process is under control at each identified CCP. Regulatory or auditing bodies commonly require the listing of each monitoring procedure and its frequency within the HACCP plan. Representative sampling of the monitoring procedure naturally will be paramount to ensuring effective monitoring. By their nature PATs monitor directly within the process stream and consequently must be non-destructive. A significant advantage of such an approach is the provision of vastly greater data sets over the traditional sampling techniques combined with a reduction of certain errors and contamination risks associated with sampling. There are numerous monitoring technologies which are currently available or offer potential as PATs for the food industry. Table 1 outlines some key technologies and their potential applications to monitor chemical, biological or physical hazards. Although principally non-destructive in nature and typically provide high temporal resolution data, ensuring effective representative sampling is critical for effective adoption of PAT. 


\begin{tabular}{|c|c|c|c|c|}
\hline \multicolumn{5}{|c|}{ Process analytical technologies for food hazards. } \\
\hline PAT & Chemical hazard & Microbial hazard & Physical hazard & Reference \\
\hline NIR spectroscopy & $\nu$ & & $\boldsymbol{\nu}$ & [13] \\
\hline Hyperspectral imaging & $\boldsymbol{\nu}$ & $\boldsymbol{\nu}$ & $\boldsymbol{\nu}$ & [14] \\
\hline Raman spectroscopy & $\nu$ & $\boldsymbol{\nu}$ & $\nu$ & [15] \\
\hline Computer vision & $\nu$ & & $\nu$ & [16] \\
\hline Magnetic resonance imaging & $\nu$ & & $\boldsymbol{\nu}$ & {$[17]$} \\
\hline Thermal imaging & & $\boldsymbol{\nu}$ & $\boldsymbol{\nu}$ & Gowen et al., 2010 \\
\hline Terahertz imaging & & & $\boldsymbol{\nu}$ & {$[18]$} \\
\hline Ultrasound & & & $\boldsymbol{\nu}$ & [19] \\
\hline Process tomography & & & $\boldsymbol{\nu}$ & [20] \\
\hline Fluorescence spectroscopy & & $\boldsymbol{\nu}$ & $\boldsymbol{\nu}$ & [21] \\
\hline Laser induced breakdown spectroscopy & $\boldsymbol{\nu}$ & レ & & {$\left[9^{\circ}\right]$} \\
\hline Electronic tongue and nose & $\nu$ & $\nu$ & & [22] \\
\hline X-ray & & & $\boldsymbol{\nu}$ & [23] \\
\hline
\end{tabular}

\section{Principle 5: Establish corrective action}

Corrective actions are actions to be taken when monitoring procedures indicate a deviation from an established critical limit. By definition PAT is a system for analysing and controlling manufacturing through timely measurements of critical quality and performance attributes of raw and in-process materials and processes. One significant benefit of PAT implementation is the potential for rapid corrective action to be taken, a key component in a successful preventative strategy. Early detection and rapid corrective responses of identified issues are desired for any manufacturing process and particularly so for products which had a safety risk.

\section{Principle 6: Verification}

Verification includes the validation of the monitoring plan (e.g., scientific support) along with demonstration that the implementation process meets the validation criteria. The application of methods, procedures tests and other evaluations, in addition to monitoring, to determine compliance with the HACCP plan. Validation can be defined as "a documented program that provides a high degree of assurance that a specific process, method, or system will consistently produce a result meeting predetermined acceptance criteria, although food regulators typically require the verification step to only address the CCP's specific to the HACCP plan. PAT tools are capable of continuously measuring product acceptance criteria and CPP's and on-line tools are capable of continuously evaluating if the process is behaving consistently. Through adoption of an effective PAT strategy the approach can provide continual process verification. Such continual process verification could offer significant assurances for the effective performance of a HACCP strategy.

\section{Principle 7: Establish documentation}

HACCP requires that there is effective documentation including hazard analysis and records documenting the monitoring of CCPs, critical limits, verification activities, and the handling of CCP deviations. A key component of PAT is effective data management and consequently dedicated software solutions have begun to emerge to handle the large and multi-variant data provided with an effective PAT strategy. Some of the advantages of using such hierarchical control system include the flexibility to input multiple analytical devices, use of data fusion techniques. Common data mining techniques are supported by such software for data pre-processing, validation, and analysis. Data mining techniques can be integrated to access required data (HACCP Principles 6, 7). Data from different sources or even from different sites could be consolidated for better product and process insights.

\section{Real-time data and smart sensing}

Advantages of adopting advanced sensors for HACCP include (1) the automation of monitoring, control and documentation, (2) provision of representative sampling from in-process monitoring, (3) early detection and response of product or processes outside of identifies critical limits, (4) potential to capture and utilise data along the complete food chain, (5) improved traceability due to increased product monitoring and (6) continuous process verification. Key to an effective HACCP strategy is an understanding of both the food product and its processing steps in terms of how they influence the identified and characterised risks. It is evident that end-product analyses provides limited information on the safety status of food, since the presence of a hazardous organism could give an indication but absence in a limited number of samples is no guarantee of safety of a whole production batch $\left[2^{\bullet \bullet}\right]$. A data rich approach should lead to increased confidence in the strategy, provide greater insights into the complexities of such hazards and ultimately deliver major benefits in public health protection. Rapid data provision can lead to faster corrective actions and also facilitate early intentions which can prevent process product reaching critical limits. 
However, dealing with large high resolution data sets brings challenges to an industry traditionally reliant on well-established sampling protocols and paper documentation. Using high resolution sensors for safety monitoring is challenging in that strategies that deal with products which are identified as out of control for brief periods during processing must be developed. A similar challenge has emerged within validated processes for pharmaceutical manufacturers where PAT strategies identify product quality fluctuations which are unlikely to be picked up by traditional sampling protocols. Data smoothing techniques are often used to overcome such issues for product quality sensing however such approaches may not be appropriate or allowed for safety monitoring or validated processes. One suggested approach could be to develop flexible decision making protocols similar to those established for microbial sampling which can take into account the product, the microorganism and the acceptable detection limits of organisms. The International Commission on Microbiological Specifications for Foods (ICMSF) proposed a preventative scheme for managing microbial risks, namely the Food Safety Objective (FSO), where a specified maximum frequency and/or concentration of a microbiological hazard in a food at the time of consumption, is deemed to provide an appropriate level of health protection. There is potential to employ PAT as a decision making protocol for determining acceptable contamination levels under the FSO initiative. MRI can be used to detect microbial activity at bacterial populations above $1000 \mathrm{cfu}$ because of the resulting chemical changes. There has been at least one commercial use of this in evaluating nutritional products. A PH drop resulting from micro organism activity was used to remove "spoiled" containers - approximately 1 per 10000 or more. As more microbiology focused PATs are developed the input of microbial expertise and regulators is required more and more.

Early adopters the framework in the pharma industry feared regulatory reprisals should PATs identify problems in a process that would not have been observed under normal process monitoring [8]. The FDA, however, recognised this concern and was quick to allay manufacturers' fears stating that manufacturers will not be penalised for doing the right thing [8].

\section{Figure 1}

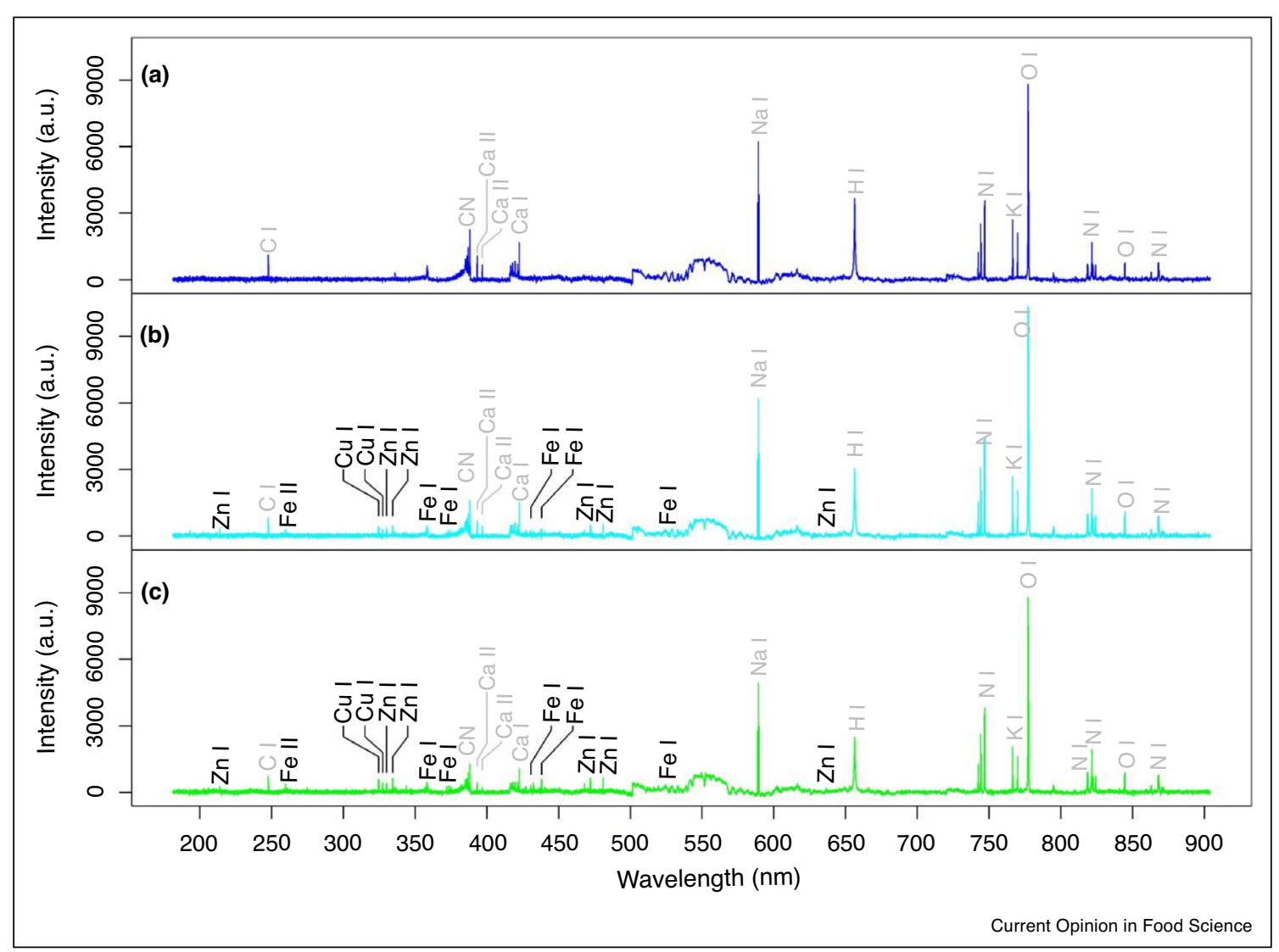

LIBS spectra of (a) pure lactose, (b) infant formula premix-lactose mixture at $50 \%$ (approx. $60 \mathrm{mg} / \mathrm{kg} \mathrm{Cu}$ and $820 \mathrm{mg} / \mathrm{kg} \mathrm{Fe}$ ) and (c) pure infant formula premix. 


\section{PAT example: laser induced breakdown spectroscopy (LIBS)}

Atomic spectroscopic techniques play a key role for food analysis. The possibility of moving such elemental analysis to the process environment would offer significant product quality and safety profiling. LIBS is a laser based technique based upon optical emission spectroscopy, where the laser pulse is absorbed by the food's surface and a small amount of material is ablated, atomised and ionised to produce a plasma plume. As the plasma cools down it emits light at a characteristic spectral structure for the elements pre-sent in the target material. A diverse range of at-, on- and in-line applications are feasible for mineral composition analysis, and also for a wide range of qualitative determinations such as authenticity, sample discrimination, bacteria contamination and elemental analysis $\left[9^{\circ}\right]$. Infant formula is a high risk food product both in regards to its compositional and microbial profile. Figure 1 shows a LIBS spectra for an infant-formula premix which could be used for mineral analysis of the product during manufacture $[10,11]$. The technique could be used for identifying product adulteration, nutrient and elemental analysis but also analysis of raw materials for heavy metals such as mercury, lead, arsenic and cadmium etc. LIBS also has the potential to identify microorganisms based on their unique atomic composition with a reference library of emission fingerprints for a wide variety of species already developed $\left[9^{\circ}\right]$. Integration of such a technology or those referenced in Table 1 have the potential to support the 7 principles of HACCP through the provision of rapid, representative and high resolution data. For example product detailed profiling of raw-materials or products could inform structured risk evaluation procedures used in HACCP principle 1. The rapid and potentially on-line nature of the technique logically supports the HACCP principles of CCP monitoring and control. The provision of high resolution product, process and risk profiling could support continuous verification and documentation.

\section{Future outlook and learning from other industries}

In the last ten years since the PAT initiative was introduced to the small molecule and biotech industries, the major regulatory agencies as well as industry have spent considerable efforts to facilitate their implementation with mixed outcomes $\left[7^{\circ}\right]$. The potential benefits in terms of product safety, control and quality are clear however real implementation is still a challenge. The food industry is typically not as limited with certain constraints such as batch production and highly validated production processes which restrain adoption of the PAT strategy. PATs are becoming increasing affordable particularly driven by developments in MEMS technology. However, there are also economical benefits to the adoption of HACCP strategies as reported by Minora and Parrett [12], who found that that the US FDA "HACCP
Procedures for the Safe and Sanitary Processing and Importing of Juice" led to significant cost savings due to reductions in the number of foodborne illnesses associated with juice-bearing products.

Knowledge of the framework in the food industry and particularly food regulators is limited and to date more focused on its application for quality assurance rather that safety. In 2009 the Food Quality, Safety \& Analysis Symposium was established at IFPAC the world's leading PAT conference to introduce the food industry to the initiative. Interest and participation from the food industry and more recently regulators have developed rapidly. Critically in terms of potential impact on HACCP there has been a significant focus on PAT for microbial and chemical safety and contamination monitoring. Rapid developments in process sensors for microbial and trace analysis will see a step change in PAT for food safety assurance and how future HACCP are implemented.

\section{References and recommended reading}

Papers of particular interest, published within the period of review, have been highlighted as:

- of special interest

$\bullet$ of outstanding interest

1. Wallace C, Holyoak L, Powell S, Dykes F: HACCP - the difficulty with hazard analysis. Food Control 2014, 35:233-240.

2. Nychas G, Panagou E, Mohareb F: Novel approaches for food - safety management and communication. Curr Opin Food Sci 2016, 12:13-20.

An excellent discussion of the potential gains of implementing PAT and information technologies in the food sector. The information provided is useful to complement this opinion piece.

3. Cullen PJ, O'Donnell C, Fagan C: Benefits and challenges of - $\quad$ adopting PAT for the food industry. In Process Analytical Technology for the Food Industry. Edited by O'Donnell FC, Fagan C, Cullen PJ. Springer Publishing; 2015. ISBN 978-1-4939-0310-8.

This publication introduces the benefits and challenges of introducing PAT strategies for food processing. The book at a whole discusses individual PATs with potential applications in the food sector.

4. Mishra A, Banerjee S, Bhatwadekar N, Mahajan P, Karode P: Process analytical technology (PAT): boon to pharmaceutical industry. Pharm Rev 2018, 6.

5. Juran JM: Juran on Quality by Design: The New Steps for Planning Quality into Goods and Services. Revised edition. New York: Free Press; 1992.

6. ICH Q8 guideline: Pharmaceutical Development, ICH Harmonised Tripartite Guideline, Step4. 2009

7. Rathore AS: QbD/PAT for bioprocessing: moving from theory - $\quad$ to implementation. Curr Opin Chem Eng 2014, 6:1-8.

This opinion piece provides an excellent discussion of the challenges and benefits of implementing a PAT strategy for the bioprocessing sector.

8. Willis R: Process Analytical Technology. Today's Chemist at Work; 2004.

9. Markiewicz-Keszycka M, Cama-Moncunill X, Casado-Gavalda M

- Dixit Y, Cama-Moncunill R, Cullen P, Sullivan C: Laser-induced breakdown spectroscopy (LIBS) for food analysis: a review. Trends Food Sci Technol 2017, 65:80-93.

This is a succinct review of LIBS as a potential PAT tool for the food industry. This work introduces the technology and discusses potential process applications.

10. Cama-Moncunill R, Casado-Gavalda M, Cama-Moncunill X, Markiewicz-Keszycka M, Dixit Y, Cullen P, Sullivan C: 
Quantification of trace metals in infant formula premixes using laser-induced breakdown spectroscopy. Spectrochim Acta B: At Spectrosc. 2017, 135:6-14.

11. Cama-Moncunill $X$, Markiewicz-Keszycka M, Dixit $Y$, CamaMoncunill R, Casado-Gavalda M, Cullen P, Sullivan C: Feasibility of laser-induced breakdown spectroscopy (LIBS) as an at-line validation tool for calcium determination in infant formula. Food Control 2017, 78:304-310.

12. Minora T, Parrett M: The economic impact of the Food and Drug Administration's Final Juice HACCP Rule. Food Policy 2017, 68:206-213.

13. Porep J, Kammerer D, Carle R: On-line application of near infrared (NIR) spectroscopy in food production. Trends Food Sci Technol 2015, 46:211-230.

14. Gowen AA, O’Donnell CP, Cullen PJ, Downey G, Frias JM: Hyperspectral imaging-an emerging process analytical tool for food quality and safety control. Trends Food Sci Technol 2007, 18:590-598.

15. Liao W, Lu X: Determination of chemical hazards in foods using surface-enhanced Raman spectroscopy coupled with advanced separation techniques. Trends Food Sci Technol 2016, 54:103-113

16. Wu D, Sun D: Colour measurements by computer vision for food quality control - a review. Trends Food Sci Technol 2013 29:5-20.
17. Mathiassen J, Misimi E, Bondø M, Veliyulin E, Østvik S: Trends in application of imaging technologies to inspection of fish and fish products. Trends Food Sci Technol 2011, 22:257-275.

18. Gowen AA, O'Sullivan C, O'Donnell CP: Terahertz time domain spectroscopy and imaging: emerging techniques for food process monitoring and quality control. Trends Food Sci Technol 2012, 25:40-46.

19. McClements DJ: Advances in the application of ultrasound in food analysis and processing. Trends Food Sci Technol 1995, 6:293-299

20. Schoeman L, Williams $\mathrm{P}$, du Plessis A, Manley M: X-ray microcomputed tomography ( $\mu \mathrm{CT}$ ) for non-destructive characterisation of food microstructure. Trends Food Sci Technol 2016, 47:10-24.

21. Cho B, Kim MS, Chao K, Lawrence K, Park B, Kim K: Detection of fecal residue on poultry carcasses by laser-induced fluorescence imaging. J Food Sci 2009, 74:E154-E159.

22. Peris M, Escuder-Gilabert L: Electronic noses and tongues to assess food authenticity and adulteration. Trends Food Sci Technol 2016, 58:40-54.

23. Nielsen MS, Lauridsen T, Christensen LB, Feidenhans'| R: X-ray dark-field imaging for detection of foreign bodies in food. Food Control 2013, 30:531-535. 